\title{
Thermodynamic origin of the slow free exciton photoluminescence rise in GaAs
}

\author{
M. Beck, J. Hübner, and M. Oestreich \\ Institut für Festkörperphysik, Leibniz Universität Hannover, Appelstraße 2, D-30167 Hannover, Germany \\ S. Bieker, T. Henn, T. Kiessling, ${ }^{\dagger}$ W. Ossau, and L. W. Molenkamp \\ Physikalisches Institut (EP3) der Universität Würzburg, 97074 Würzburg, Germany
}

(Received 2 June 2015; published 5 February 2016)

\begin{abstract}
We use time-resolved photoluminescence (TRPL) spectroscopy to unequivocally clarify the microscopic origin of the nanosecond free exciton photoluminescence rise in GaAs at low temperatures. In crucial distinction from previous work, we examine the TRPL of the GaAs free exciton second LO-phonon replica. This enables us to simultaneously monitor the unambiguous time evolution of the total exciton population and the cooling dynamics of the initially hot free exciton ensemble. We demonstrate by a model based on the Saha equation and the experimentally determined cooling behavior that the long-debated slow photoluminescence rise is caused by time-dependent shifts in the thermodynamic quasiequilibrium between free excitons and the uncorrelated electron-hole plasma.
\end{abstract}

DOI: 10.1103/PhysRevB.93.081204

Introduction. Time-resolved photoluminescence spectroscopy is widely used to investigate the kinetics of free exciton formation, relaxation, and recombination in semiconductors and their nanostructures [1]. In most experiments performed at low lattice temperatures a significantly delayed onset of the free exciton luminescence with respect to the excitation laser pulse is observed [2-13]. This slow photoluminescence (PL) rise has attracted intense research interest for nearly three decades. However, a general consensus on its microscopic origin has still not been reached.

Clearly, an unequivocal study of the time evolution of a photoexcited free exciton ensemble is challenging. Typical time-resolved photoluminescence (TRPL) experiments use ultrafast above-band-gap optical excitation and monitor the time evolution of the initially hot photocarrier cloud by the emission intensity of the free exciton zero-phonon line $(F X)$. However, because of momentum conservation during the luminescence process, only a subset of the entire free exciton population close to the Brillouin zone center can recombine radiatively [14-16]. In particular, hot excitons, which exhibit large $K$ vectors, are not visible in the zero-phonon line. The emission intensity of the $(F X)$ zero-phonon line is hence not a direct measure of the actual free exciton density $n_{X}(t)$ in the crystal, a fact which is a major obstacle to the unambiguous interpretation of such $(F X)$ TRPL signals.

We therefore assess in this Rapid Communication the time evolution of an optically excited free exciton ensemble by TRPL traces detected on the free exciton second LO-phonon replica $(F X)-2 \hbar \Omega_{\mathrm{LO}}$. The strict $K$ vector selection rule applicable to the $(F X)$ zero-phonon line is fully relaxed for the radiative annihilation of free excitons under simultaneous emission of two LO phonons. This is because the wave vectors of the two LO phonons can always add up such that they compensate for the center-of-mass momentum of the recombining exciton $[17,18]$. In contrast to previous TRPL studies of the $(F X)$ zero-phonon line, we here trace the actual

\footnotetext{
*jhuebner@nano.uni-hannover.de

†tobias.kiessling@physik.uni-wuerzburg.de
}

time evolution of the free exciton density, which is not distorted by the relaxation of large $K$ vector excitons to the radiative zone. Moreover, a time-resolved line shape analysis of the $(F X)-2 \hbar \Omega_{\mathrm{LO}}$ replica provides direct experimental access to the exciton cooling curve $T_{X}(t)$.

Having directly determined the cooling dynamics from experiment, we next address the impact of the exciton cooling on the transient thermodynamic quasiequilibrium between free excitons and unbound electron-hole pairs, which is fundamentally described by the Saha equation. It has previously been shown that the Saha equation allows for a precise description of the spatially dependent population balance of the partially ionized exciton gas under continuous-wave laser excitation in bulk [19] and of the time evolution of the exciton gas in semiconductor quantum wells [7,20]. We here demonstrate that the experimental free exciton TRPL traces in bulk GaAs, and in particular the slow photoluminescence rise, is also consistently described by the shift of the thermodynamic ionization equilibrium from the uncorrelated electron-hole plasma (EHP) to the free exciton state.

Sample and experimental setup. Our study aims at the investigation of the undisturbed time evolution of a free exciton population. Even in lightly doped samples, repopulation processes among defect-induced carrier reservoirs crucially influence the dynamics of the exciton ensemble [21,22]. To avoid such spurious influences on the free exciton TRPL traces, we investigate a piece of nearly defect-free GaAs. The sample is a nominally undoped, $1.5-\mu \mathrm{m}$-thick epilayer of (001)-oriented molecular-beam-epitaxy-grown GaAs. The active layer is sandwiched between a 250 period $\mathrm{GaAs} / \mathrm{Al}_{0.09} \mathrm{Ga}_{0.91} \mathrm{As}$ superlattice on the bottom and a 80 period superlattice of equal composition on top to prevent optically excited excitons from diffusing out of the layer and to suppress surface recombination [23]. The low-temperature PL spectrum of the sample (Fig. 1) is dominated by the free exciton recombination line $(F X)$, which indicates a negligible density of impurity atoms in the active layer. From a comparison with spectra reported in the literature [10,24], we estimate a residual impurity concentration of $\lesssim 1 \times 10^{12} \mathrm{~cm}^{-3}$. Polariton effects are neglected on the basis of the small L-T splitting in bulk 


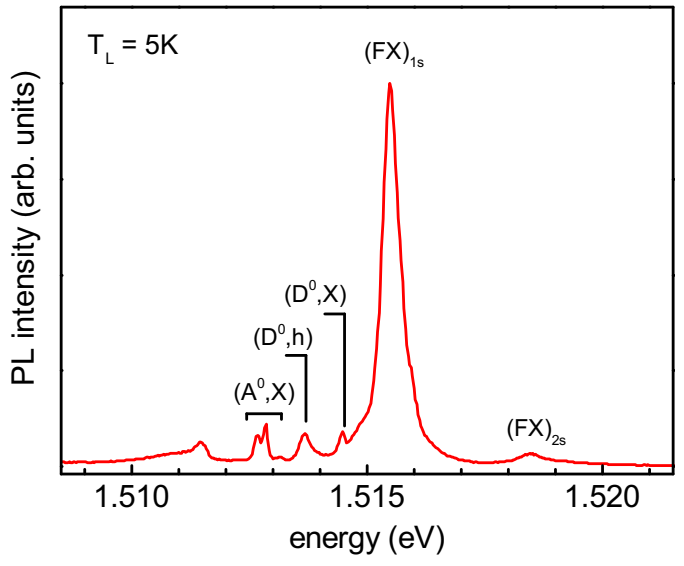

FIG. 1. The continuous wave excitation low-temperature PL spectrum of the investigated sample is dominated by free exciton luminescence $(F X)$, which attests a negligible impurity concentration in the active GaAs layer. Text labels indicate the usual nomenclature of free and bound exciton transitions [27].

GaAs compared with the homogeneous linewidth of the free exciton resonance $[15,25,26]$.

The sample is held at $T_{L}=5 \mathrm{~K}$ lattice temperature in a liquid helium continuous-flow optical cryostat. Optical excitation at $\lambda_{\text {exc }}=780 \mathrm{~nm}$ is provided by a pulsed Ti:sapphire laser. The pulse width of $\approx 2 \mathrm{ps}$ is determined from autocorrelation measurements. To prevent measurement artifacts originating from previous excitation pulses [12], the repetition rate is reduced to $4 \mathrm{MHz}$ by a pulse picker (extinction ratio $<1: 1500$ ). To mitigate spurious influences of lateral carrier diffusion, the laser beam is only moderately focused on the sample surface by a $f=200 \mathrm{~mm}$ achromatic lens to a $\left(1 / e^{2}\right)$ intensity spot diameter of $95 \mu \mathrm{m}$, which significantly exceeds the free exciton diffusion length of approximately $10 \mu \mathrm{m}$ in our sample [28]. For the carrier diffusion coefficient $D \approx 70 \mathrm{~cm}^{2} \mathrm{~s}^{-1}$ [28], the diameter of the Gaussian profile expands only by $\approx 12 \%$ within the first $5 \mathrm{~ns}$ after the excitation pulse, during which the carrier energy relaxation and the exciton luminescence rise take place. The change in carrier density due to diffusive dilution is much weaker than the concurrent reduction of the photocarrier density by radiative recombination. It therefore has only a negligible impact on the time evolution of the carrier ensemble and is consequently neglected in our later modeling. The luminescence is dispersed in a $250 \mathrm{~mm}$ focal length imaging spectrometer equipped with a $1200 \mathrm{~mm}^{-1}$ grating. Time-resolved PL spectra are detected by a streak camera equipped with a two-dimensional charge-coupled device.

Exciton cooling. Pulsed off-resonant optical excitation creates an initially hot photocarrier population, which cools towards $T_{L}$ by emission of optical and acoustic phonons and simultaneously dilutes by concurrent radiative decay $[1,29]$. Since the free exciton PL onset is crucially influenced by the transient photocarrier cooling, which governs the dynamic equilibrium between free excitons and unbound charge carriers, we first consider the exciton cooling curve $T_{X}(t)$.

Previous works have determined the transient cooling of optically excited hot electron populations from a time-resolved

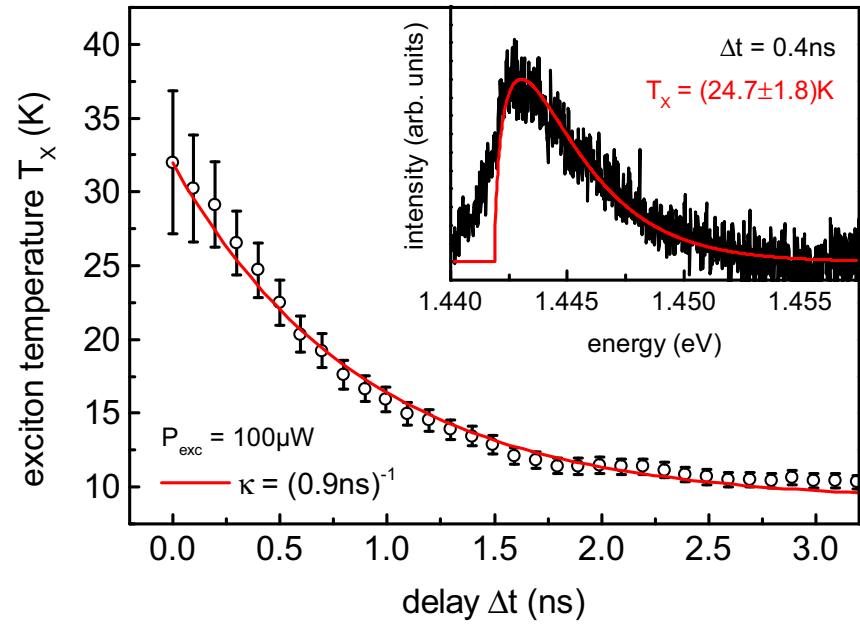

FIG. 2. Transient cooling of the nonresonantly excited exciton population. Data points are obtained from Maxwellian line shape analyses of the $(F X)-2 \hbar \Omega_{\mathrm{LO}}$ phonon replica. The solid red curve indicates a Newtonian cooling with a time constant $\kappa=(0.9 \mathrm{~ns})^{-1}$. Recombination heating prevents full equilibration of the photocarrier ensemble with the crystal lattice. A representative spectrum and Maxwellian line shape fit to the $(F X)-2 \hbar \Omega_{\mathrm{LO}}$ replica is shown in the inset.

line shape analysis of the free-electron to neutral-acceptor transition $\left(e, A^{0}\right)$ [30]. On the contrary, the temperature of a free exciton ensemble is not readily obtained from the luminescence line shape of the $(F X)$ zero-phonon line and we are not aware of any previous study that reports experimental exciton cooling curves in high-purity GaAs. The exciton temperature, however, can be obtained from a spectral analysis of the $(F X)$ second LO-phonon replica, which mirrors the kinetic energy distribution function of the free exciton population [17,18]. A time-resolved Maxwellian line shape analysis of the weak $(F X)-2 \hbar \Omega_{\mathrm{LO}}$ transition therefore provides direct experimental access to the transient temperature of the hot exciton ensemble [31].

A representative cooling curve $T_{X}(t)$ at $P_{\text {exc }}=100 \mu \mathrm{W}$ excitation power is shown in Fig. 2. The monotonic decrease of the exciton temperature is well described by straightforward Newtonian cooling

$$
\frac{d}{d t} T_{X}=-\kappa\left(T_{X}-T_{0}\right)
$$

with a cooling constant $\kappa=(0.9 \mathrm{~ns})^{-1}$ and a final exciton temperature $T_{0}=9 \mathrm{~K}$, which is higher than the lattice temperature.

Compared with the peculiar temperature dependence of the electron cooling rates [30], the assumption of a constant exciton cooling rate $\kappa$ allows for a surprisingly good approximation of the actual cooling curve $T_{X}(t)$ for $9 \mathrm{~K} \leqslant T_{X} \leqslant 32 \mathrm{~K}$. We note that the exciton ensemble relaxes its excess energy more efficiently than one would expect from Ref. [30] for a mere electron population. We speculate that the faster excess energy dissipation of free excitons reflects the hole contribution to the cooling process, for which $\approx 2.5 \times$ larger cooling rates have been reported [1]. 
The fact that $T_{0}=9 \mathrm{~K}$ does not coincide with the lattice temperature $T_{L}=5 \mathrm{~K}$ is a consequence of momentum conservation during the luminescence process. From the thermalized free exciton ensemble with a Boltzmann kinetic energy distribution, only the subset of excitons at $K \approx 0$ recombines radiatively $[15,16]$. Therefore the luminescence process selectively removes low-energy excitons from the entire ensemble. The remaining kinetic energy is redistributed by scattering processes among the leftover excitons, whose temperature (in the absence of competing cooling channels) thus increases. The selective radiative decay of low-energy excitons at $K \approx 0$ effectively serves as a heating mechanism for the exciton population. At long delay times, this "recombination heating" compensates the cooling rate due to acoustic phonon emission and accounts for the discrepancy between $T_{0}$ and $T_{L}$ [32,33].

For the purpose of our later model description, we verified that the the cooling constant $\kappa$ and the final exciton temperature $T_{0}$ do not depend on excitation density. The starting temperature of the cooling curve is individually determined for each excitation power $P_{\text {exc }}$ [34].

Buildup of a free exciton population. We show in Fig. 3(a) the time-resolved emission intensity, i.e., the spectrally integrated PL emission of the second LO-phonon replica, at varying excitation densities. In agreement with previous TRPL studies of the $(F X)$ zero-phonon line, we observe a significantly delayed PL onset with respect to the excitation laser pulse. The delay is most pronounced in the low excitation limit. For increasing excitation power $P_{\text {exc }}$, the PL maximum gradually shifts to shorter delay times.

As a main result, our measurements unambiguously demonstrate that the delayed $(F X)$ PL onset observed in previous studies is not mainly caused by the relaxation of large $K$ vector excitons to the Brillouin zone center. The slow PL rise of the $(F X)-2 \hbar \Omega_{\mathrm{LO}}$ replica, which monitors the total exciton density $n_{X}(t)$, rather demonstrates a relatively slow buildup of the free exciton population.

The population balance between Coulomb-bound free excitons and the uncorrelated EHP is described by the Saha equation $[35,36]$

$$
\frac{n_{e} n_{h}}{n_{X}}=\left(\frac{k_{B} T_{X}}{2 \pi \hbar^{2}}\right)^{3 / 2}\left(\frac{m_{e} m_{h}}{m_{X}}\right)^{3 / 2} \exp \left(-\frac{E_{B}}{k_{B} T_{X}}\right)
$$

with $n_{e}, n_{h}$, and $n_{X}$ representing the electron, hole, and free exciton densities and $m_{e}, m_{h}$, and $m_{X}$ the electron, hole, and free exciton effective masses, respectively [37]. $E_{B}=4.2 \mathrm{meV}$ denotes the exciton binding energy in bulk GaAs [27] and $T_{X}$ is the exciton temperature. With the substitutions $n_{X}=f_{X} n_{0}$ and $n_{e}=n_{h}=\left(1-f_{X}\right) n_{0}$, the Saha equation allows one to determine the relative fraction $f_{X}$ of free excitons in a photocarrier population of total pair density $n_{0}$ at a given temperature $T_{X}$.

The thermodynamics of the partially ionized exciton gas is illustrated in Fig. 3(d) by a contour plot $f_{X}\left(n_{0}, T_{X}\right)$. The population balance between free excitons and the EHP is crucially influenced by both the exciton temperature $T_{X}$ and the total photocarrier density $n_{0}$. Thermal breakup of free excitons into pairs of unbound charge carriers causes a decrease of the exciton population for increasing temperatures. At fixed temperature, however, an increase of the photocarrier pair density $n_{0}$ raises the fraction of free excitons.

In the framework of our model, we expect the strongest delay in the PL rise for the highest initial carrier excess
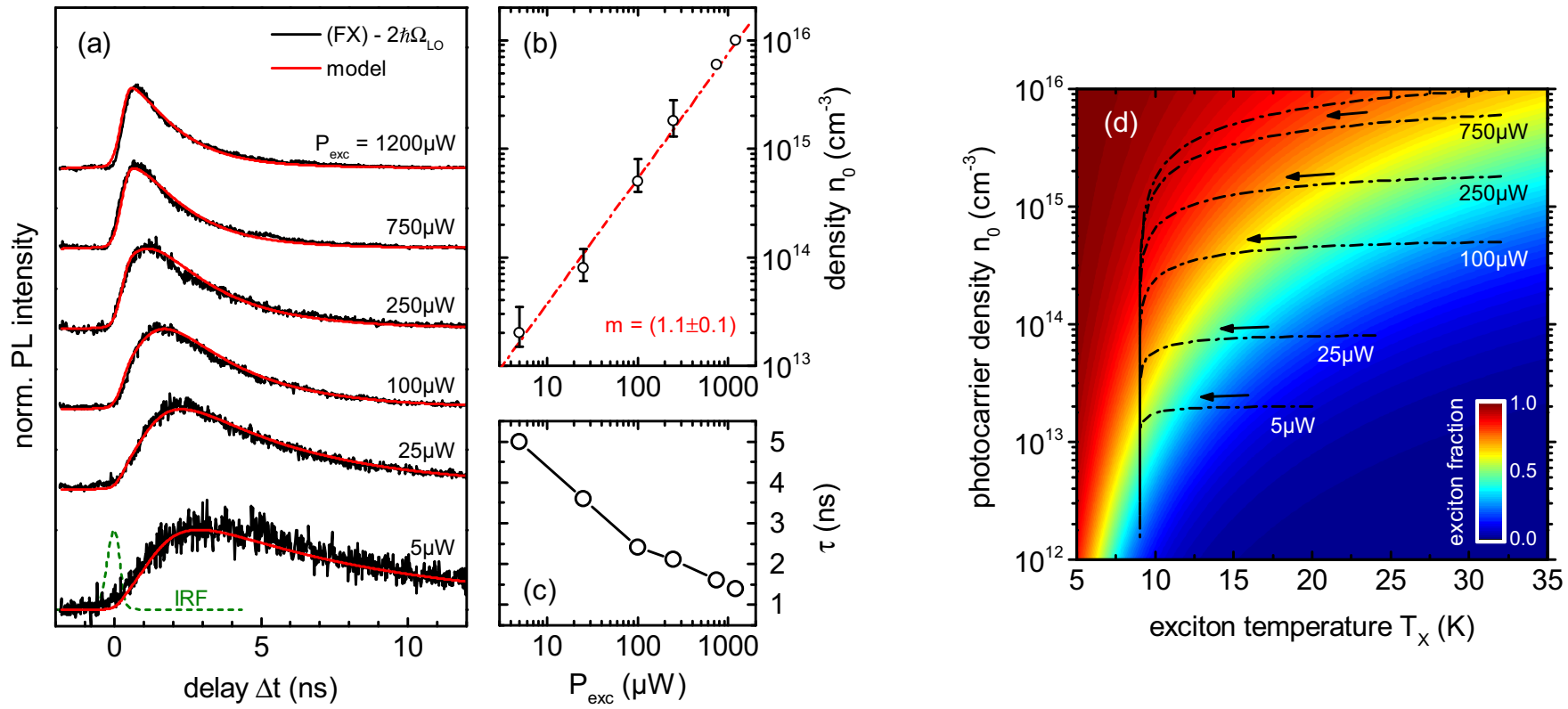

FIG. 3. (a) Excitation power dependence of the PL time traces detected on the free exciton second LO-phonon replica $(F X)-2 \hbar \Omega_{\mathrm{LO}}$. Solid red lines are our model results. (b) The total photocarrier density per excitation pulse extracted from our model scales linearly with the time-averaged optical excitation density $P_{\text {exc }}$. We note that the PL onset at $P_{\text {exc }}=750 \mu \mathrm{W}$ and $1200 \mu \mathrm{W}$ is dominated by the width of the IRF. We therefore disregard the respective data points for the power law fit. (c) Decay times $\tau$ used in the model. (d) Contour plot of the thermodynamic equilibrium of the partially ionized exciton gas described by the Saha equation. Color indicates the exciton fraction $f_{X}=n_{X} / n_{0}$ in a photocarrier population of total pair density $n_{0}$. Exciton formation is thermodynamically favored at high photocarrier densities and low temperatures. Dash-dotted lines indicate the trajectories of the model time traces shown in (a). 
temperature. We therefore perform all measurements at the lowest accessible lattice temperature $T_{L}=5 \mathrm{~K}$.

To derive a conceptual model that describes the time evolution of the free exciton density $n_{X}(t)$ after pulsed optical excitation, we consider the interplay of transient cooling and decay of the entire photocarrier ensemble. The link between $T_{X}(t)$ [Eq. (1)], $n_{0}(t)$, and $n_{X}(t)$ is provided by the Saha equation (2).

As time elapses, the photocarrier ensemble dilutes by concurrent free exciton and EHP recombination. For the sake of simplicity, we neglect the weaker bimolecular EHP recombination (cf. the negligible emission intensity from direct band-to-band transitions in Fig. 1) and assume that $n_{0}(t)$ decays exclusively by the much more efficient channel of free exciton radiative decay $[13,38]$. The time evolution of the photocarrier pair density $n_{0}(t)$ is then described by the rate equation

$$
\frac{d}{d t} n_{0}=-\frac{n_{0} f_{X}\left(n_{0}, T_{X}\right)}{\tau}
$$

with the ensemble lifetime $\tau$. From the Saha equation (2), the time evolution of the free exciton density $n_{X}(t)$ is related to the pair density $n_{0}(t)$ as

$$
n_{X}(t)=n_{0}(t) f_{X}\left(n_{0}, T_{X}\right) .
$$

A comparison of our conceptual model with the experimental $n_{X}(t)$ time evolution obtained from the $(F X)-2 \hbar \Omega_{\mathrm{LO}}$ replica is shown in Fig. 3(a). For each excitation density, we numerically solve the coupled equations (1)-(4) and fit the convolution of the calculated $n_{X}(t)$ with the measured instrument response function (IRF) to the $(F X)-2 \hbar \Omega_{\mathrm{LO}}$ time traces. Excellent agreement with the experiment is obtained by only adjusting the photocarrier density $n_{0}(t=0)$, i.e., the number of photoexcited electron-hole pairs per excitation pulse and the ensemble lifetime $\tau$.

The initial pair density $n_{0}(t=0)$ obtained from our model analysis is plotted in Fig. 3(b) as a function of the timeaveraged excitation power $P_{\text {exc }}$. We regard the linear scaling of the density $n_{0}(t=0)$ with $P_{\text {exc }}$ as strong evidence that our model captures the essential physics governing the time evolution of the free exciton population [39]. The lifetimes $\tau$ used in our model are reported in Fig. 3(c). We note that efficient exciton-exciton and exciton-free carrier scattering cause a broadening of the $(F X)$ zero-phonon line of width $\Delta E$, which increases as a function of the excitation density [40]. Since only excitons in the radiative zone with energy $E \leqslant$ $\Delta E$ recombine radiatively $[15,16]$, the ensemble lifetime $\tau$ expectedly decreases for increasing excitation densities. It is worth noting that this observation is not in conflict with the relaxed $K$ vector selection rule of the second LO-phonon replica analyzed by us: because of the inherent weakness of the $(F X)-2 \hbar \Omega_{\mathrm{LO}}$ transition, the time evolution of the exciton population is still completely governed by the orders of magnitude stronger $(F X)$ zero-phonon recombination which depends on $\Delta E$.
To provide an intuitive picture of the long PL rise times and to illustrate the temporal shift of the $(F X)$ PL maximum as a function of the optical excitation power, we show in Fig. 3(d) the trajectories of the TRPL traces [i.e., our model results for $\left.n_{X}(t)\right]$ in the Saha phase diagram. At low and medium excitation powers, the EHP is thermodynamically favored at early delays because of the pronounced photocarrier overheating. The peak exciton density only occurs after the photocarrier ensemble has sufficiently cooled such that the population balance shifts from the EHP towards the Coulomb-bound excitons. After a certain delay, the maximum absolute exciton density $n_{X}(t)$ is reached, although the total photocarrier density $n_{0}(t)$ decreases monotonically after the laser pulse. At high excitation powers, despite the high initial carrier temperature, exciton formation is still already thermodynamically favored at early delays because of the high photocarrier pair density $n_{0}$. Therefore, we consistently observe an earlier arrival of the exciton PL maximum in the experiment.

As a final test of consistency, we tune the laser resonantly to the $(F X)$ zero-phonon line. Under such resonant optical excitation, we observe an instantaneous (i.e., undelayed) PL onset. This result further confirms our model, because no excess energy is provided to the exciton ensemble, i.e., $T_{X}(t=$ $0)=T_{0}$ and transient carrier cooling does not take place.

Summary and conclusion. We have performed TRPL measurements of the free exciton second LO-phonon replica emission in bulk GaAs to trace the undistorted time evolution of a nonresonantly excited free exciton population. Our study unambiguously demonstrates that the delayed $(F X)$ PL onset is not caused by momentum relaxation of large $K$ vector excitons to the radiative zone, but rather by the transient electron-hole pair to free exciton interconversion described by the less frequently considered Saha equation. Throughout the investigated excitation density range, our conceptual model obviates consideration of the recently proposed Coulombcorrelated EHP state [41,42].

The Saha equation also predicts the existence of free excitons at $k_{B} T_{L} \geqslant 4.2 \mathrm{meV}$ (the exciton binding energy) and therefore provides a natural alternative explanation to the formation of a Coulomb-correlated EHP for the presence of free exciton luminescence at elevated sample temperatures $T_{L} \geqslant 48 \mathrm{~K}$ [13].

Moreover, our study directly reveals that the cooling dynamics of a hot free exciton ensemble in bulk GaAs is crucially affected by recombination heating. Even after long delay times, the exciton population does not fully equilibrate with the crystal lattice. This finding potentially has detrimental implications for the search of excitonic Bose-Einstein condensates, which require the preparation of ultracold free exciton ensembles.

Acknowledgments. The excellent GaAs sample was grown by J. J. Harris and C. T. Foxon at Philips Research Laboratories, Redhill, UK. The authors gratefully acknowledge financial support by the DFG (SPP1285 OS98/9-3, GRK 1991) and the EU (ERC-AG project 3-TOP).
[1] J. Shah, Ultrafast Spectroscopy of Semiconductors and Semiconductor Nanostructures (Springer, Berlin, 1999).
[2] J.-i. Kusano, Y. Segawa, Y. Aoyagi, S. Namba, and H. Okamoto, Phys. Rev. B 40, 1685 (1989). 
[3] T. C. Damen, J. Shah, D. Y. Oberli, D. S. Chemla, J. E. Cunningham, and J. M. Kuo, Phys. Rev. B 42, 7434 (1990).

[4] R. Eccleston, R. Strobel, W. W. Rühle, J. Kuhl, B. F. Feuerbacher, and K. Ploog, Phys. Rev. B 44, 1395 (1991).

[5] P. Roussignol, C. Delalande, A. Vinattieri, L. Carraresi, and M. Colocci, Phys. Rev. B 45, 6965 (1992).

[6] T. Amand, B. Dareys, B. Baylac, X. Marie, J. Barrau, M. Brousseau, D. J. Dunstan, and R. Planel, Phys. Rev. B 50, 11624 (1994).

[7] H. W. Yoon, D. R. Wake, and J. P. Wolfe, Phys. Rev. B 54, 2763 (1996).

[8] M. Gulia, F. Rossi, E. Molinari, P. E. Selbmann, and P. Lugli, Phys. Rev. B 55, R16049 (1997).

[9] R. Höger, E. Göbel, J. Kuhl, K. Ploog, and H. J. Quiesser, J. Phys. C: Solid State Phys. 17, L905 (1984).

[10] J. Aaviksoo, I. Reimand, V. V. Rossin, and V. V. Travnikov, Phys. Rev. B 45, 1473 (1992).

[11] M. Gurioli, P. Borri, M. Colocci, M. Gulia, F. Rossi, E. Molinari, P. E. Selbmann, and P. Lugli, Phys. Rev. B 58, R13403(R) (1998).

[12] I. Reimand and J. Aaviksoo, Phys. Rev. B 61, 16653 (2000).

[13] A. Amo, M. D. Martin, L. Viña, A. I. Toropov, and K. S. Zhuravlev, Phys. Rev. B 73, 035205 (2006).

[14] S. Permogorov, Phys. Status Solidi B 68, 9 (1975).

[15] G. W. 't Hooft, W. A. J. A. van der Poel, L. W. Molenkamp, and C. T. Foxon, Phys. Rev. B 35, 8281 (1987).

[16] J. Feldmann, G. Peter, E. O. Göbel, P. Dawson, K. Moore, C. Foxon, and R. J. Elliott, Phys. Rev. Lett. 59, 2337 (1987).

[17] E. Gross, S. Permogorov, and B. Razbirin, J. Phys. Chem. Solids 27, 1647 (1966).

[18] B. Segall and G. D. Mahan, Phys. Rev. 171, 935 (1968).

[19] S. Bieker, T. Henn, T. Kiessling, W. Ossau, and L. W. Molenkamp, Phys. Rev. Lett. 114, 227402 (2015).

[20] R. A. Kaindl, D. Hägele, M. A. Carnahan, and D. S. Chemla, Phys. Rev. B 79, 045320 (2009).

[21] O. Brandt, J. Ringling, K. H. Ploog, H.-J. Wünsche, and F. Henneberger, Phys. Rev. B 58, R15977 (1998).

[22] F. Münzhuber, T. Henn, T. Kiessling, W. Ossau, L. W. Molenkamp, B. Gieseking, G. V. Astakhov, and V. Dyakonov, Phys. Rev. B 90, 125203 (2014).

[23] R. J. Nelson and R. G. Sobers, J. Appl. Phys. 49, 6103 (1978).

[24] U. Heim and P. Hiesinger, Phys. Status Solidi B 66, 461 (1974).

[25] L. Schultheis, J. Kuhl, A. Honold, and C. W. Tu, Phys. Rev. Lett. 57, 1797 (1986)
[26] M. Cardona and G. Güntherodt, Light Scattering in Solids III. Recent Results (Springer-Verlag, Berlin, 1982).

[27] M. R. Brozel and G. E. Stillman, Properties of Gallium Arsenide (INSPEC, London, 1996).

[28] S. Bieker, T. Kiessling, W. Ossau, and L. W. Molenkamp, Phys. Rev. B 92, 121201(R) (2015).

[29] S. A. Lyon, J. Lumin. 35, 121 (1986).

[30] R. Ulbrich, Phys. Rev. B 8, 5719 (1973).

[31] D. Hägele, R. Zimmermann, M. Oestreich, M. R. Hofmann, W. W. Rühle, B. K. Meyer, H. Amano, and I. Akasaki, Phys. Rev. B 59, R7797(R) (1999).

[32] D. Bimberg and J. Mycielski, Phys. Rev. B 31, 5490 (1985).

[33] A. L. Ivanov, J. Phys.: Condens. Matter 16, S3629 (2004).

[34] For excitation densities $P_{\text {exc }}>100 \mu \mathrm{W}$, the $(F X)-2 \hbar \Omega_{\mathrm{LO}}$ line shape at short delays is severely distorted by the spectrally nearby LO-phonon replica of the donor-acceptor pair transition $\left(D^{0}, A_{C}^{0}\right)-\hbar \Omega_{\mathrm{LO}}$, which impedes a robust determination of the starting temperature. From the peculiar temperature dependence of the cooling rates [30], we assume $T_{X}(0) \approx 32 \mathrm{~K}$ for $P_{\text {exc }}>$ $100 \mu \mathrm{W}$.

[35] W. D. Kraeft, K. Kilimann, and D. Kremp, Phys. Status Solidi B 72, 461 (1975)

[36] G. Bastard, Wave Mechanics Applied to Semiconductor Heterostructures (Halsted Press, New York, 1988).

[37] In bulk GaAs, $m_{e}=0.067 m_{0}$ and $m_{h}=0.51 m_{0}$ with $m_{0}=$ $9.11 \times 10^{-31} \mathrm{~kg}$ the free electron mass and $m_{X}=m_{e}+m_{h}$.

[38] J. Szczytko, L. Kappei, J. Berney, F. Morier-Genoud, M. T. Portella-Oberli, and B. Deveaud, Phys. Rev. Lett. 93, 137401 (2004).

[39] The degeneracy factors of the involved photocarrier species (electrons, holes, and free excitons) are not precisely known, which impedes a quantitative comparison of the model pair density $n_{0}(t=0)$ with the number of photoexcited charge carriers per excitation pulse estimated from the laser repetition rate and the excitation volume. However, the degeneracy factors only rescale $n_{0}(t=0)$, but do not depend on excitation parameters. We therefore expect the model pair density to scale linearly with the optical excitation power $P_{\text {exc }}$, which we demonstrate in Fig. 3(b).

[40] L. Schultheis, J. Kuhl, A. Honold, and C. W. Tu, Phys. Rev. Lett. 57, 1635 (1986).

[41] M. Kira, F. Jahnke, and S. W. Koch, Phys. Rev. Lett. 81, 3263 (1998).

[42] S. W. Koch, M. Kira, G. Khitrova, and H. M. Gibbs, Nat. Mater. 5, 523 (2006) 